\title{
A Polynomial Fitting Improved Bayesian Reconstruction Method for Whole Brain Volumetric MRSI Metabolite Images
}

\author{
Yufang Bao ${ }^{1, *}$ and Andrew Maudsley ${ }^{2}$ \\ ${ }^{I}$ Department of Mathematics and Computer Science/Center of Defense and Homeland Security (CDHS), UNC Fayette- \\ ville State University, NC, USA \\ ${ }^{2}$ Department of Radiology, Miller School of Medicine, University of Miami, FL, USA
}

\begin{abstract}
In this paper, a polynomial fitting improved Bayesian approach is proposed for the reconstruction of volumetric metabolite images from long echo time (TE) whole brain proton magnetic resonance spectroscopic imaging (MRSI) data. The proposed algorithm uses a modified EM (expectation maximization) algorithm that takes into account the partial volume effects contained inside a thick slice MRSI. It incorporates high resolution volumetric magnetic resonance imaging (MRI) as a priori information. It further integrates the polynomial fitting method to smooth out artificial edges before the high resolution metabolite images are reconstructed. Our proposed reconstruction method has successfully extended our existing reconstruction of two dimensional (2D) metabolite images to 3D cases. The experimental results show that resolution enhanced volumetric metabolite images are reconstructed.
\end{abstract}

Keywords: MRSI $k$-space data, volumetric metabolite images, Bayesian image reconstruction, polynomial fitting.

\section{BACKGROUND}

In vivo magnetic resonance spectroscopy imaging (MRSI) is a non-invasive method to measure valuable metabolite concentrations in the human brain [1-15]. The MRSI data consist of four dimensional complex valued spectral data that can be used to locate the spatial concentrations of various metabolites inside the brain. Each metabolite concentration is identified at its unique resonance frequency of protons that is characterized by the spectral component of the MRSI data. Typically, a sub-sampling scheme is used for MRSI acquisition to shorten imaging time due to the long scan time required for collecting a large MRSI dataset. Image reconstruction methods are usually applied off-line in order to retrieve various metabolite concentrations, particularly, to obtain high resolution metabolite images from MRSI. In this paper, we propose a mathematical modeling and polynomial fitting improved Bayesian reconstruction method to obtain resolution enhanced 3D metabolite images from whole brain MRSI.

\subsection{Characteristics Of MRSI Data}

The metabolite images reconstructed from one single slice MRSI are so far of poor quality. The primary concern is that the MRSI data are characterized by very low spatial resolution. The data are accompanied by weak signal-to-noise ratio (SNR) due to low metabolite concentrations in the human brain. This results in the large voxel size used in

*Address correspondence to this author at the Department of Mathematics and Computer Science, UNC Fayetteville State University, Fayetteville, NC 28301; Tel: 910-672-2437(o); Fax: 910-672-1070;

E-mail: ybao@uncfsu.edu.
MRSI studies. A typical voxel has 5-mm to $10-\mathrm{mm}$ in-plane size and its slice thickness is about $10-\mathrm{mm}$ to $15-\mathrm{mm}$. This is relatively large when compared to the small voxel size used in volumetric MRI studies, namely, $0.5-\mathrm{mm}$ in-plane size and 3-mm slice thickness. Another concern is the distortion in the acquired MRSI signals caused by patient motion during a prolonged acquisition of MRSI. Although the usage of low resolution in the whole brain volumetric MRSI studies has shortened the scanning time, it still takes about 30 minutes. In addition, the data are contaminated by the magnetic field inhomogeneity, which causes strong signal distortion in certain regions. These factors together have led to the difficulty in reconstructing high resolution metabolite images.

\subsection{Image Reconstruction with MRSI Data}

The brain metabolites of interest in MRSI are Nacetylaspartate (NAA), total choline (Cho), and total creatine $(\mathrm{Cr})$. Several studies have concluded that changes in metabolite concentrations can be used as indicators for the presence of tumors/cancers [16-18]. A straightforward way to generate the metabolite images is to estimate the intensity of each metabolite voxel-by-voxel, and then integrate the fitted intensities from all voxels together as images. However, it results in poor image quality because the metabolite signals are of low resolution, and the data contain artifacts, such as the Gibbs ringing effects that come from the residues of strong signals, such as water and lipid. Therefore, it is necessary to reconstruct high resolution metabolite images using MRI $a$ priori information.

Methods for reconstructing metabolite images from 2D MRSI data was initiated in the early 1990s [3-10]. The SLIM (spectral localization by imaging) [8] and its generalized method GSLIM (generalized SLIM) [9] are based on the 
assumption that the spectra confined to an anatomical compartment are homogeneous. The GSLIM are designed to capture some spectral variation within each compartment. Further, SLOOP (spectral localization with optimal point spread function) [10] proposes to optimize the phaseencoding gradients, and to characterize the regions of interest by matching the point spread functions. Later, a spatial frequency inhomogeneity correction [11] is used following the SLIM-type algorithm method. A Bayesian statistical reconstruction method [1] is proposed using a modified EM algorithm to incorporate the anatomical partial volume tissue concentrations from registered high resolution MRI images as a priori information. This algorithm is further tuned to process the clinical data. Significant improvement has been demonstrated for single slice MRSI. Several methods have been further proposed [19-21] to improve reconstruction using high resolution MRI as a priori. Recently, the line shapes derived from magnetic field mapping [22] are used as references for reconstructing metabolite images, and a sparse spectral model incorporated MRI a priori information [23] is used to overcome artifacts and to minimize the noise presented in the MRSI data.

For 3D MRSI data, the existing reconstruction methods may be directly used to reconstruct metabolite images along the in-plane $(x y)$ direction on a slice-by-slice basis. This reconstruction can be repeated along $x z$ or $y z$ direction to reduce overlaid metabolite concentrations in the through slice direction; however, this will lead to accumulated reconstruction errors. In this paper, we propose an algorithm that extends our 2D statistical Bayesian approach to 3D metabolite image reconstruction. Our new approach reconstructs high resolution metabolite images simultaneously in all three spatial directions. Our Bayesian reconstruction incorporates the highresolution a priori information provided by the volumetric MRI, and it further integrates the polynomial fitting method to reconstruct resolution enhanced metabolite images.

\section{METHODOLOGY}

\subsection{The Mathematical Modeling of Whole Brain Volu- metric MRSI Data}

The $k$-space data of the long TE whole brain MRSI are assumed to fit the mathematical model described in Eqn. (2.1), where $S\left(k_{x}, k_{y}, k_{z}, t\right)$ is a complex-valued free induction decay (FID) MRSI signal at $k$-space location $k_{x}, k_{y}, k_{z}$, with time $t$. For each spatial location $(x, y, z), A_{m}(x, y, z)$ is the spatial signal intensity from the $m$ th $^{\text {th }}$ metabolite (or the intensity is proportional to its actual concentration); $T_{\mathrm{a}}$ and $T_{\mathrm{b}}$ are two parameters that characterize the spatial Lorentzian and Gaussian line shape components in the FID signals; $\Delta B_{0}$ is the deviation of the magnetic field from the ideal field strength $B_{0} ; \varnothing_{0}$ is the $0^{\text {th }}$-order phase term; $f_{\mathrm{m}}$ is the resonance frequency generated by the $m^{\text {th }}$ metabolite; $\gamma$ is the gyromagnetic ratio constant, and $j$ stands for the unit imaginary number. In this paper, long TE MRSI of the human brain are considered because most heavy molecules have tended to fade down at this stage, and typically only the three major singlet resonances of three metabolites of interest, namely, NAA, Cho, Cr, are present in the MRSI signals apart from a baseline for each $(x, y, z)$. The imaging object is assumed to be of high resolution with dimensions $N_{x}, N_{y}$, and $N_{z}$.
Volumetric echo planar spectroscopic imaging (EPSI) [12-15] is used in whole brain volumetric MRSI acquisition. Initially, the raw spatial data points acquired from 3D EPSI do not match the linear spatial locations; rather, the data follow paths that can be typically described as sinusoidal functions. Hence, the raw MRSI data are re-sampled to correct the sampling trajectory so that the resulting MRSI data properly reflect the spatial distance. After re-sampling, the MRSI data can be described by Eqn. (2.1). This model is built for equally spaced MRSI signals based on the Block equation.

$$
\begin{aligned}
& S\left(k_{x}, k_{y}, k_{z}, t\right)=\sum_{m=1}^{M} \sum_{x=0}^{N_{x}-1} \sum_{y=0}^{N_{y}-1} \sum_{z=0}^{N_{z}-1} A_{m}(x, y, z) e^{-\frac{t}{T_{a}(x, y, z)}-\frac{t^{2}}{T_{b}(x, y, z)}} \\
& e^{\left\{-j 2 \pi\left[f_{m}(x, y, z)+\gamma \Delta B_{0}(x, y, z)\right] t+\varphi_{0}(x, y, z)+\left(\frac{x k_{x}}{N_{x}}+\frac{y k_{y}}{N_{y}}+\frac{z k_{z}}{N_{z}}\right)\right\}}
\end{aligned}
$$

The acquired data usually span only a small portion of the "central" $k$-space with dimensions $K_{x}, K_{y}, K_{z}$, with $K_{x}<$ $N_{x}, K_{y}<N_{y}$, and $K_{z}<N_{z}$. The "central" $k$-space data are sampled at $\left(k_{x}, k_{y}, k_{z}\right)$, with $k_{x}=-K_{x} / 2$ to $K_{x} / 2, k_{y}=-K_{y} / 2$ to $K_{y} / 2$, and $k_{z}=-K_{z} / 2$ to $K_{z} / 2$. The observed data, $d\left(k_{x}, k_{y}, k_{z}, t\right)$, are composed of the metabolite signals, $S$, the baseline signals, $b$, and the noise signals, $n$, following the statistical model in $k$-space as described in Eqn. (2.2).

$$
d\left(k_{x}, k_{y}, k_{z}, t\right)=S\left(k_{x}, k_{y}, k_{z}, t\right)+b\left(k_{x}, k_{y}, k_{z}, t\right)+n\left(k_{x}, k_{y}, k_{z}, t\right)
$$

The baselines are typically assumed to be smooth and slowly varied curves. They cannot be parameterized because of their complexity, but they can be mostly removed from the data in a pre-reconstruction procedure. Therefore, the baselines are neglected from Eqn. (2.2) so that we can focus on the reconstruction method. The noise is assumed to be independent and identically distributed (i.i.d.) Gaussian noise with zero mean and variance $\sigma^{2}$.

Our reconstruction method aims to estimate the missing high spatial-frequency content of the MRSI data to reach the desired dimensions, $N_{x}, N_{y}, N_{z}$. Note that the existing reconstruction methods deal with single slice image reconstruction, namely, $N_{z}=1$. For our volumetric data reconstruction, we assume $K_{z}<N_{z}$. Our optimization method simultaneously takes spatial MRI as a priori. This reduced the fuzzy effect (see Fig. 1) introduced by the overlaid metabolite concentrations in the through slice direction. Subsequently, the metabolite images with relatively thinner slice thickness will be reconstructed with enhanced features.

\subsection{Improved Reconstruction Algorithm for Volumetric MRSI Data}

In this section, the EM algorithm is used to estimate the metabolite intensities using Eqn. (2.3), assuming that $d\left(k_{x}, k_{y}, k_{z}, t\right)-S\left(k_{x}, k_{y}, k_{z}, t\right)=n\left(k_{x}, k_{y}, k_{z}, t\right)$ are i.i.d. normal distributions. The a priori information from the volumetric $M R I$ provides some knowledge of the metabolite intensity $A_{m}(x, y, z)$ that is contained in the signal $S\left(k_{x}, k_{y}, k_{z}, t\right)$, as described in Eqn. (2.1). Note that there is a connection be- 

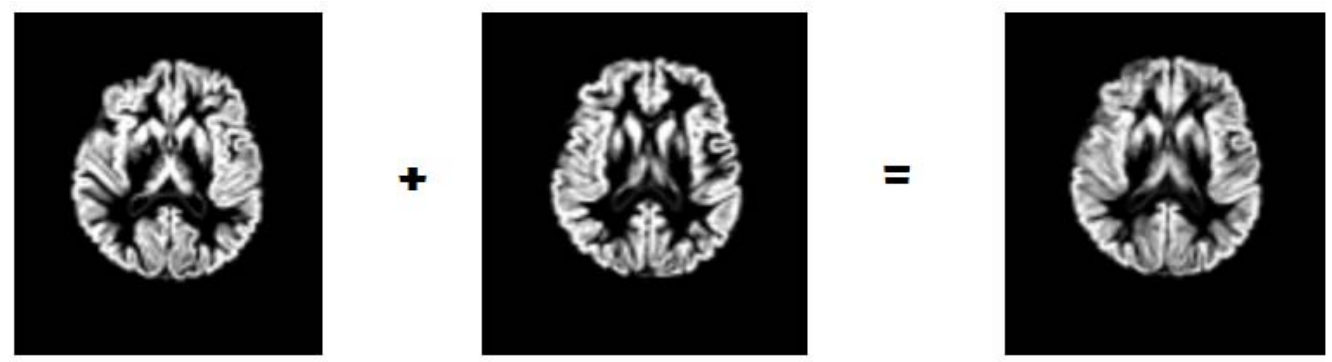

Fig. (1). Through-slice partial volume effects: In the left, two thin slices of gray matter images were overlaid, resulting in a thick slice image of gray matter in the right hand side. Partial volume effect can be observed in the thick slice gray matter image.
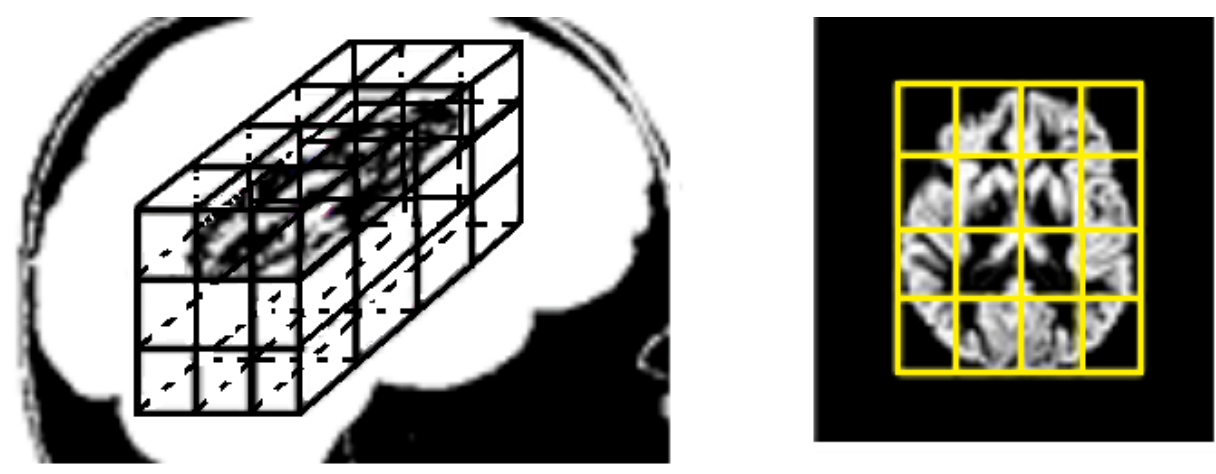

Fig. (2). The sub-volumes of the human brain that are corresponding to a single thick slice MRSI were defined using three thin image slices. As a result, three thin slices MRSI metabolite image of high resolution can be reconstructed.

tween the MRI and MRSI studies. The two imaging formats are originated from the same anatomical structure of the human brain, because the MRI data are typically collected immediately prior to the acquisition of the MRSI data. However, they have different resolutions. The volumetric MRI images demonstrate high-resolution anatomical structure of the brain with some significant features that may not exist in the MRSI. The a priori knowledge of volumetric MRI in Eqn. (2.3) can be replaced by the fraction of tissue concentrations, such as gray matter (GM), white matter (WM), CSF (CSF), and lipid (LIP), in a desired high-resolution setting to reconstruct the intensity of each metabolite for every voxel from the MRSI data.

The $V_{u}$ in Eqn (2.4) represents the partial volume segmentation for a given tissue ' $u$ '. It describes the percentage of the $u^{\text {-th }}$ tissue contributed to each voxel at the desired volumetric resolution. It is obtained using the following steps:

1. The high-resolution volumetric MRI images are segmented to obtain tissue distributions of GM, WM, CSF, and LIP using SPM segmentation program [24], which incorporates both T1 and T2 volumetric MRI images.

2. The high-resolution tissue volumetric distributions (in the $x, y, z$ directions) are co-registered to all voxels inside the MRSI data to estimate the metabolite contributions originated from each tissue.

$$
\hat{A}_{m}(x, y, z)=\underset{A_{m}(x, y, z)}{\operatorname{argmax}} P\left(\left\{d\left(k_{x}, k_{y}, k_{z}, t\right)\right\} \mid \text { Volumetric MRI }\right)
$$

$\hat{A}_{m}(x, y, z)=\underset{A_{m}(x, y, z)}{\arg \max } \mathrm{P}\left(\left\{d\left(k_{x}, k_{y}, k_{z}, t\right)\right\} \mid V_{G M}(x, y, z), V_{W M}(x, y, z), V_{C S F}(x, y, z), V_{L I P}(x, y, z)\right)$

\section{Modified EM Algorithm}

It is observed that the metabolite concentrations are naturally homogeneous within a pure tissue in a small localized volume; therefore, an efficient way for reconstructing MRSI data is to further divide each volumetric tissue into subvolumes at the desired-resolution. The $3 \mathrm{D}$ volume inside the imaging FOV is cut into cubes that are referred to as subvolumes as shown in Fig. (2). The percentage of each tissue inside a sub-volume is defined in Eqn. (2.5), in which $n$ is the total number of sub-volumes used for each tissue.

A sub-volume is said to have low tissue concentration when $p_{u, r}<$ threshold, where the threshold is set at $10 \%$. A sub-volume with low tissue concentration is merged into its neighboring sub-volume (the one with the lowest concentration among the neighboring sub-volumes except the one with consideration) to form a larger sub-volume. This allows each sub-volume to include a significant amount of tissue. As a result, smaller amount of parameters are to be estimated. This is done for GM, WM, CSF, and LIP separately, and a varied total number of sub-volumes, denoted as $R_{u}$, may be resulted for each tissue type $u$; therefore, the selection of sub-volumes depends on each tissue type. Fig. (2) shows an example of sub-volume selection for GM. Therefore, Eqn. (2.4) can be replaced by Eqn. (2.6), in which $V_{u, r}$ is subvolume for each tissue, $u$ is the tissue type, and $r, r=1,2, \cdots, R_{u}$, is the sub-volume index. It is further assumed that the metabolite concentrations are slowly varying in space, thus could be considered as constant across every 
sub-volume for each individual tissue type. Eqn. (2.6) is then reformulated into Eqn. (2.7) assuming constant metabolite intensities, denoted as $b_{\text {mur }}$, over $r^{\text {th }}$ sub-volume, $u^{\text {th }}$ tissue, and for the $m^{\text {th }}$ metabolite, namely, NAA, Cho, and Cr. Meanwhile, Eqn (2.1) can be approximated using Eqn. (2.8), where $F P_{V_{u, r}}$ is the $m^{\text {th }}$ spectral signal constrained to subvolume $V_{u, r}$ as in Eqn. (2.9).

$p_{u, r}=\frac{\text { \#voxels of } u^{-t h} \text { tissue in } r^{-t h} \text { volume }}{\text { \#total voxels in } r^{-t h} \text { voume }}$,
$r=1, \cdots, n_{u}$, for each $u=G M, W M, C S F, L I P$ estimate the average metabolite intensities using Eqn. (2.10), in which $R Z$. satisfies Eqn. (2.11).

The $R Z\left(m, u, r, k_{x}, k_{y}, k_{z}, t\right)$ are the $k$-space signals that combine the metabolite intensities, the metabolite spectra, and are contaminated by Gaussian noise, $n_{\text {mur }}^{R Z}\left(k_{x}, k_{y}, k_{z}, t\right)$, as shown in $R Z$. The $T v_{\mathrm{u}, \mathrm{r}}(x, y, z)$ is the percentage of $u^{-t h}$ tissue at $(x, y, z)$ within sub-volume $v_{\mathrm{u}, \mathrm{r}}$. Based on this adjustment of the parameters, our modified EM algorithm is obtained by iterating the E-step and the M-step in Eqns. (2.12) and (2.13)

The initial value of $b_{m u r}$ is set to zero. Iterating the E-step

$\hat{A}_{m}(x, y, z)=\underset{A_{m}(x, y, z)}{\arg \max } \mathrm{P}\left(\left\{d\left(k_{x}, k_{y}, k_{z}, t\right)\right\} \mid V_{G M, r}(x, y, z), V_{W M, r}(x, y, z), V_{C S F, r}(x, y, z), V_{L I P, r}(x, y, z)\right)$

$\hat{b}_{\text {mur }}=\underset{b_{\text {mur }}}{\operatorname{argmax}} \mathrm{P}\left(\left\{d\left(k_{x}, k_{y}, k_{z}, t\right)\right\} \mid V_{G M, r}, V_{W M, r}, V_{C S F, r}, V_{L I P, r}\right)$

$S_{b}\left(k_{x}, k_{y}, k_{z}, t\right)=\sum_{m=1}^{M} \sum_{u=1}^{N} \sum_{r=1}^{R_{u}} b_{m u r} F P_{V_{u, r}}\left(k_{x}, k_{y}, k_{z}, t, m\right)$

$F P_{V_{u, r}}\left(k_{x}, k_{y}, k_{z}, t, m\right)=\sum_{(x, y, z) \in V_{u, r}} e^{-\frac{t}{T_{a}(x, y, z)}-\frac{t^{2}}{T_{b}(x, y, z)}}$

$e^{\left\{-j 2 \pi\left[f_{m}(x, y, z)+\gamma \Delta B_{0}(x, y, z)\right] t+\varphi_{0}(x, y, z)+\left(\frac{x k_{x}}{N_{x}}+\frac{y k_{y}}{N_{y}}+\frac{z k_{z}}{N_{z}}\right)\right\}}$

$d\left(k_{x}, k_{y}, k_{z}, t\right) \approx S_{b}\left(k_{x}, k_{y}, k_{z}, t\right)+n\left(k_{x}, k_{y}, k_{z}, t\right)=$

$\sum_{m=1}^{M} \sum_{u=1}^{N} \sum_{r=1}^{R_{u}} R Z\left(m, u, r, k_{x}, k_{y}, k_{z}, t\right)$

$R Z=b_{\text {mur }} \sum_{x=0}^{N_{x}} \sum_{y=0}^{N_{y}} \sum_{z=0}^{N_{z}} T_{V_{u, r}}(x, y, z) V_{u r}(x, y, z) f_{m}(x, y, z) e^{-j 2 \pi\left(\frac{k_{x}}{N_{x}}+\frac{k_{y}}{N_{y}}+\frac{k_{z}}{N_{z}}\right)}$

$+n_{\text {mur }}^{R Z}\left(k_{x}, k_{y}, k_{z}, t\right)$

with

$$
\begin{aligned}
& f m(x, y, z)=e^{-\frac{t}{T_{a}(x, y, z)}-\frac{t^{2}}{T_{b}(x, y, z)}} \\
& e^{\left\{-j 2 \pi\left[f_{m}(x, y, z)+\gamma \Delta B_{0}(x, y, z)\right] t+\varphi_{0}(x, y, z)\right\}}
\end{aligned}
$$

The E-step:

$$
R Z^{\text {new }}\left(m, u, r, k_{x}, k_{y}, k_{z}, t\right)=E\left(\left\{R Z\left(m, u, r, k_{x}, k_{y}, k_{z}, t\right)\right\} \mid d, b^{\text {old }}\right)
$$

The M-step:

$$
b_{\text {mur }}^{\text {new }}=\underset{b_{\text {mur }}}{\arg \max } P\left(\left\{R Z^{\text {new }}\right\} \mid S_{b}\left(k_{x}, k_{y}, k_{z}, t\right)\right)
$$

The average sub-volume metabolite intensities for different tissues are then estimated iteratively using the proposed signal model and the estimated spectral parameters. The EM algorithm is modified based on the one proposed in [1] to and the M-step results in our estimation, $b_{\text {mur }}^{\text {new }}$. The iteration terminates when $\left|b_{m u r}^{\text {new }}-b_{m u r}^{\text {old }}\right|<\varepsilon$, typical $\varepsilon=0.001$. The estimated metabolite intensity, $b_{\text {mur }}$, is the average density of the $m^{\text {th }}$ metabolite, in the sub-volume, $V_{u, r}$. The estimated parameter, $b_{m u r}$, is further combined with the fraction of each tissue to reconstruct each metabolite distribution and is used in the further processing in the following section.

\section{Polynomial Fitting}

The average metabolite signal intensities from all tissue sub-volumes are estimated using the modified EM-algorithm described in Eqns. (2.12) and (2.13). The amount of voxels contained in each 3D sub-volume is multiple times greater than the amount of pixels contained in a $2 \mathrm{D}$ sub-region. Thus, significant different values between two adjacent subvolumes are resulted from estimation due to the large amount of voxels used in estimating the average metabolite intensity of a sub-volume. As a result, the metabolite images reconstructed directly from the EM algorithm demonstrates significant contrast between two neighboring locations. This is what we called the blocky patch phenomenon. Additional processing steps are needed to avoid the artifacts being carried over to the metabolite images reconstructed at the final stage. Since we assume the metabolite intensities follow a smooth and slowly changing pattern within each pure tissue, it is sufficient for us to selectively apply polynomial fitting to the reconstructed intermediate metabolite images, denoted as $I_{b}(x, y, z)$. Note that the assumption may be somehow limited as recent research has shown evidence of significant change in some areas inside the brain [25]; our reconstruction method indeed relatively enhances such significant change as extrapolation is used in our reconstruction.

The polynomial fitting of an intermediate metabolite image is performed in the 2D plane for each $z$ of higher resolution, according to Eqn. (2.14).

$$
I_{U}^{n}(x, y, z)=\min _{f^{n}} \sum_{(x, y) \in U}\left[f^{n}(x, y, z)-I_{b}(x, y, z)\right],
$$

where $f^{n}(x, y, z)$ is a 2D polynomial function of degree $n$, for fixed $z$. Indeed, each image plane, $I_{b}(x, y, z)$, is fitted to a 
broaden region, $U$, including the sub-volume boundary. The region $U$ is obtained using the morphological operation for dilation. Polynomial function of degree 2 is typically used in the polynomial fitting, and it is shown to be more robust than using polynomials of higher degrees according to experiments.

\section{MRSI Data Extrapolation and Metabolite Image Recon- struction}

After the polynomial fitting is applied, the reconstructed intermediate metabolite images, $I_{U}^{n}(x, y, z)$, are then combined with the estimated spectral parameters to reconstruct the MRSI data of dimensions $N_{x}, N_{y}, N_{z}$, using Eqn. (2.8). The spatial high-frequency data that correspond to the missing data locations are selected and extrapolated to the lowresolution volumetric MRSI data to form a new set of highresolution volumetric MRSI data using Eqn. (2.15). Inverse Fourier transformation in the spatial domain and Fourier transformation in the time domain are subsequently applied to the reconstructed high-resolution MRSI data.

$$
\begin{gathered}
\tilde{S}\left(k_{x}, k_{y}, k_{z}, t\right)=\left\{\begin{array}{r}
S\left(k_{x}, k_{y}, k_{z}, t\right), \\
S_{b}\left(k_{x}, k_{y}, k_{z}, t\right),
\end{array}\right. \\
\text { for }\left|k_{x}\right|<\frac{N_{x}}{2},\left|k_{y}\right|<\frac{N_{y}}{2},\left|k_{z}\right|<\frac{N_{z}}{2} \\
\text { elsewhere }
\end{gathered}
$$

Since MRSI collects spectral signals from all the voxels in the brain, the spectral fitting package [2] is used to retrieve the desired metabolite intensities voxel-by-voxel to form metabolite images. The advantage of our proposed algorithm is that it simultaneously incorporates the volumetric anatomical edge information into Bayesian method to estimate the intensities of the metabolite concentrations. It incorporates the spectral information of each metabolite provided by the MRSI, and it preserves the original MRSI spectra in the low frequency $k$-space domain.

The usage of sub-volumes has simplified the mathematical model of the MRSI and hence has simplified the reconstruction of 3D metabolite images by reducing the number of parameters needed to be estimated. It has subsequently improved the estimate of the metabolite intensities in all spatial dimensions. The reconstructed metabolite images have thinner slice thickness and each slice has higher in-plane resolution. It also reduced the memory load that puts a huge demand on the computational resource in 3D cases. In addition, this reconstruction method also practically avoids potential underestimate problem when the EM algorithm is applied, namely, it avoids the problem of insufficient sample size in a typical voxel-by-voxel estimate method, in which a large amount of voxel intensities needed to be estimated for each metabolite.

\section{Supplementary Methods}

Our proposed algorithm assumes that the signal model is a good fit for the data; however, in practice, the in vivo MRSI data persistently contain unwanted signals, distorted signals, noise, as well as catastrophic signals due to magnetic field distortion. Typical unwanted signals include, but not limit to, the water and lipid signals, which are much stronger than the metabolite signals of interest, and additional signal components, such as baselines that cannot be effectively modeled. The distorted signals are referred to metabolite signals that are usually distorted due to eddy current effects. The catastrophic signals are those strongly distorted signals in the boundary regions, such as the air-filled sinuses in the inferior frontal lobes and the inferior temporal lobes, where high and low susceptible materials have induced local magnetic field change. These signals cause mismatch between the MRSI and its mathematical model; therefore these useless signals contained in the MRSI data must be either removed or must be restored before the MRSI data can be used for reconstruction.

Additional steps, such as pre-acquisition steps and prereconstruction steps, must be applied to the MRSI to regularize the signals and prepare the data for the model. The preacquisition steps include improvements in the pulse sequence design to optimize the useful signals in the acquisition steps, which is beyond our research scope. The prereconstruction steps are regulating the acquired MRSI before our reconstruction method is applied. The pre-reconstruction steps include improvements, such as baseline removals, strong signal removals, eddy current corrections, and subregional bad signal removals, as discussed in [1]. The prereconstruction procedure is essential for volumetric MRSI reconstruction to ensure our model fits the MRSI data.

\section{EXPERIMENTAL RESULTS AND DISCUSSIONS}

The algorithm was first applied to simulated volumetric MRSI data to reconstruct metabolite images, and the output matches well with our expectation. It is then applied to in vivo MRSI data. The experimental results from both simulated data and in vivo volumetric MRSI are demonstrated in Fig. (4) and Fig. (5). The results from the simulated MRSI clearly showed improved reconstruction. Since the reconstruction steps are similar for both datasets, here only the in vivo MRSI reconstruction is discussed.

\subsection{Materials and Methods}

A volumetric MRSI dataset from one healthy female volunteer at age 38 was studied. In the acquisition, one set of volumetric high-resolution MRI was obtained immediately prior to the MRSI acquisition using a head coil (data can be acquired using parallel MRI technique; it is omitted here as this is not our focus in this paper). All the data were obtained using a 3 Tesla clinical whole body scanner (Siemens Healthcare). The parameters for MRSI acquisition are $\mathrm{TE}=70-\mathrm{ms}$, $\mathrm{TR}=1710-\mathrm{ms}$, and $\mathrm{TI}=198-\mathrm{ms}$. The MRSI data have $50 \mathrm{x} 50 \mathrm{x}$ $18 k$-space points, 256 time-domain data, with a $280-\mathrm{mm} \mathrm{x}$ $280-\mathrm{mm}$ FOV, resulting in voxel size of $5.6-\mathrm{mm}$ in the $x y$ plane, and $10-\mathrm{mm}$ slice thickness in $z$ direction. The MRI data have $512 \times 512$ points in each slice, with $256-\mathrm{mm} \times 256-\mathrm{mm}$ FOV, resulting in $0.5-\mathrm{mm} \times 0.5-\mathrm{mm}$ in-plane resolution, and $1-\mathrm{mm}$ slice thickness, and a total of 144 slices.

Tissue concentrations were obtained through segmentation performed on the high-resolution volumetric MRI data using the SPM package [24]. The tissue concentrations were co-registered to the spatial locations and the FOV of the 


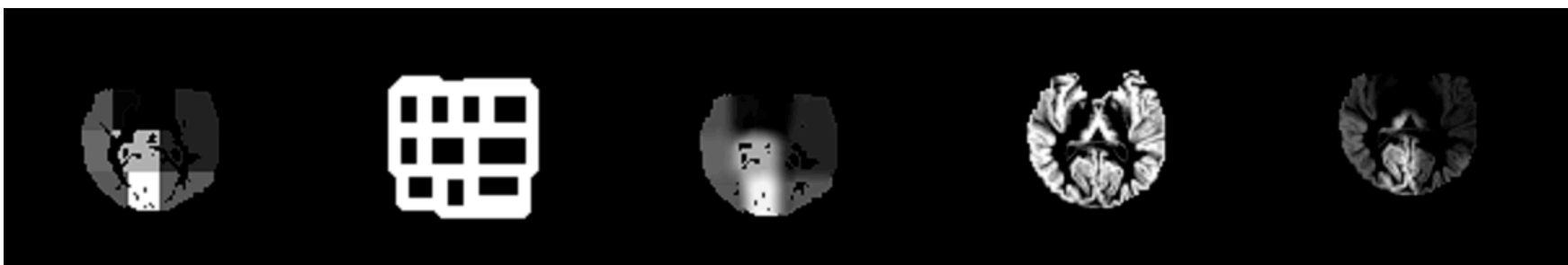

(a)

(b)

(c)

(d)

(e)

Fig. (3). The polynomial fitting procedure following the EM algorithm is shown here. (a). The NAA image constrained to sub-volumes in the gray matter is reconstructed. (b) The region where the polynomial fitting is performed. (c). The NAA image reconstructed after polynomial fitting. (d). The partial volume tissue concentration for the gray matter is used as a priori. (e). The NAA metabolite constrained to the gray matter.

(i)

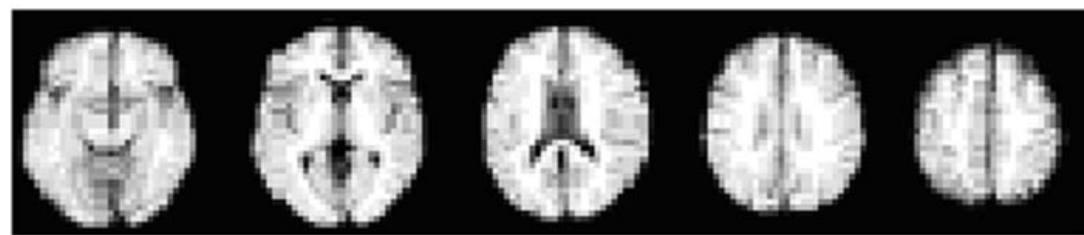

(ii)

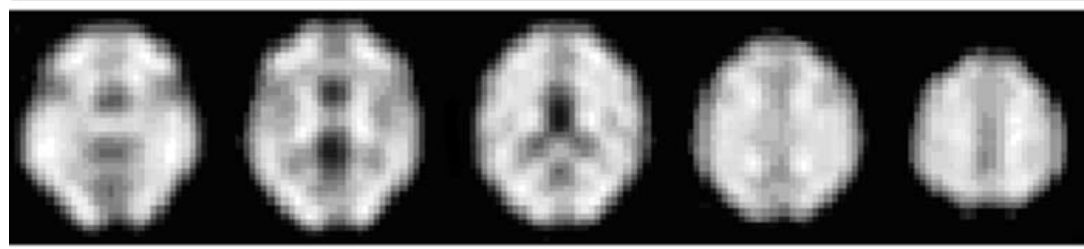

(iii)

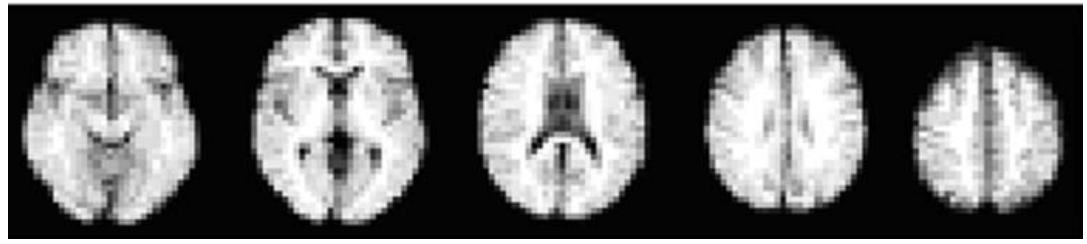

Fig. (4). Multiple slices of Cho images simulated are shown in the row (i), the reconstructed Cho image from zero-filled FT of $k$-space MRSI data are shown in row (ii), and the reconstructed metabolite images using the proposed are shown in row (iii). Note that the reconstructed metabolite images in each column are corresponded to the same slice position.

MRSI, and then re-sampled to the desired resolution with $128 \times 128 \times 36$ spatial points. High resolution MRSI data were reconstructed using our proposed reconstruction method, to reconstruct MRSI data with the in-plane spatial dimensions of $128 \times 128$ points, 36 slices, and 256 timedomain points.

In the experiment, the whole brain MRI images were divided into $4 \times 8 \times 3$ sub-volumes; then those sub-volumes without tissue concentrations were discarded, while those sub-volumes with low tissue concentrations were merged to their neighboring sub-volumes. Our algorithm, which combined the modified EM optimization algorithm with polynomial fitting, was applied to reconstruct the metabolite intensities. A new MRSI dataset, with voxel size of 2-mm in the xy-plane and 5-mm slice thickness, was further reconstructed. The volumetric metabolite images of NAA, Cr, and Cho, of enhanced resolution, were finally quantified.

\subsection{Results}

Fig. (3) displays some images showing the procedure to reconstruct the NAA metabolite distribution in the gray matter. The intermediate NAA image (shown in Fig. 3a) was reconstructed before the polynomial fitting procedure was applied. The image has shown some blocky artifacts. The sub-volume pattern (shown in Fig. 3b) was used for our reconstruction, and it showed the broadened boundary area where a polynomial fitting was applied. A smooth NAA metabolite image obtained after the polynomial fitting is shown in Fig. (3c). The partial volume tissue distribution of the gray matter (shown in Fig. 3d) is used in metabolite reconstruction. The polynomial fitted metabolite image of a pure tissue was weighted by its partial volume concentrations to generate a metabolite image that reflected the metabolite concentrations in this tissue. The NAA distribution in the gray matter is shown in Fig. (3e).

The metabolite images for other metabolites (Cho, and $\mathrm{Cr})$ were obtained in a similar manner. All metabolite images were combined with the estimated frequency information to reconstruct the high-frequency data of the MRSI $k$-space (include the time-domain data). The data were then extrapolated to the regularized low resolution $k$-space MRSI to form the MRSI data at the desired spatial resolution. Automatic spectral fitting was then applied to obtain the final metabolite images. Show in Fig. (4) is the reconstructed Cho images for the simulated data, and shown in Fig. (5) is the reconstructed NAA metabolite images. 
(i)

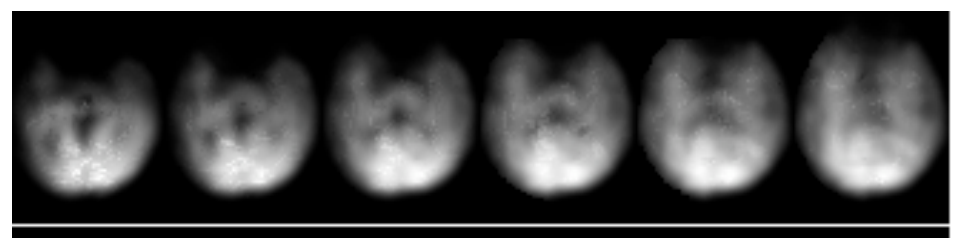

(ii)

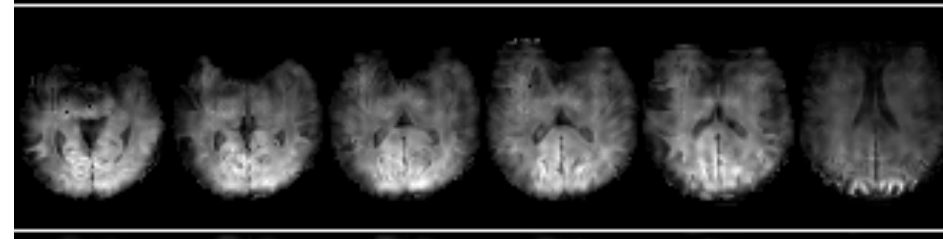

(iii)

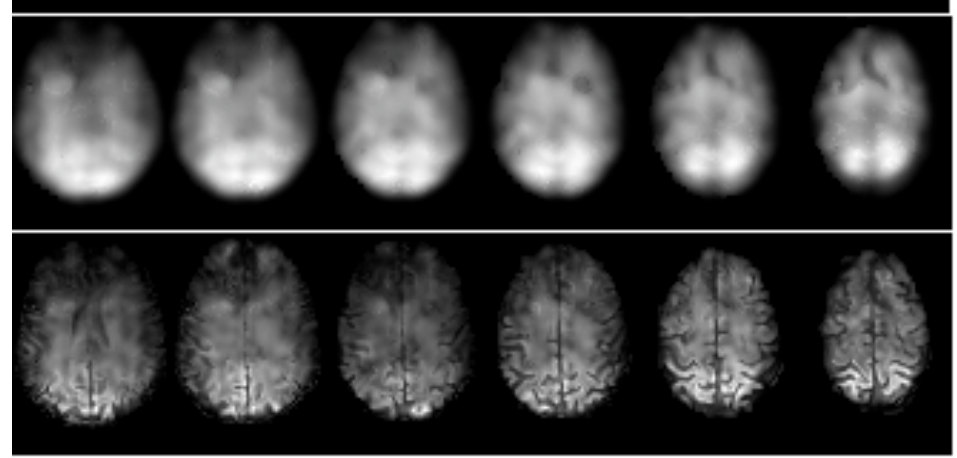

Fig. (5). Multiple slices of NAA images reconstructed from zero-filled FT of the in vivo volumetric $k$-space MRSI data (row (i) and (iii)), and using the proposed algorithm (row (ii) and (iv)). Note that the reconstructed metabolite image is of higher spatial resolution in all spatial dimensions, comparing to the original acquisition.

This study has proposed a volumetric reconstruction algorithm that uses a priori high-resolution spatial information to reconstruct the metabolite images to high spatial resolution. The algorithm incorporates the polynomial fitting and has resulted in an improved reconstruction of the metabolite images with higher resolution in $x y$-plane and thinner slice thickness. The results shown in Fig. (4) have demonstrated significant improvement in row (iii) when compared to the zero-filled FT reconstruction in row (ii), while the simulated Cho metabolite images are shown in row (i). Some slices of the reconstructed NAA metabolite images from in vivo MRSI data are shown in Fig. (5) (row (ii) and row (iv)); for comparison, the conventional zero-filled FT reconstructed NAA metabolite image slices are shown as well in Fig. (5) (row (i) and row (iii)). The images reconstructed with our proposed algorithm have demonstrated enhanced resolution in all the spatial directions.

\subsection{Discussions}

Improved structural features were demonstrated in the metabolite images reconstructed using our proposed algorithm when compared to those reconstructed using zerofilled Fourier transform (FT). In the zero-filled FT reconstruction, spatial Gaussian filters were used to reduce strong Gibbs ringing effects in the volumetric metabolite images; therefore, some over-smooth can be observed in the resultant metabolite images. The metabolite images reconstructed using our proposed algorithm have avoided this problem, and the enhanced metabolite images have clearly shown the CSF regions. In addition, higher metabolite intensities in posterior brain regions can be observed in the enhanced metabolite images.

Using our proposed algorithm, the metabolite images with increased resolution are obtained. This is resulted from extrapolating the missing high frequency content to the low resolution MRSI, which is achieved by combining our modified EM algorithm and the polynomial fitting method. The polynomial fitting ensures that the artifacts from the intermediate metabolite images (as shown in Fig. 3) were suppressed so that they were not being carried over to the final stage. The output from our algorithm has demonstrated better visibility of the metabolite intensities comparing to the output from the existing reconstruction method.

It is necessary to mention that a few metabolite image slices corresponding to the top and to the bottom of the brain in the through slice direction were discarded for the in vivo MRSI. This is due to the very poor quality data components corresponding to these regions. This is the result of a) the susceptible magnetic gradient in these regions due to quick change in the magnetic field, and b) the problem of misalignment between MRI and MRSI in these regions due to FOV change and possible patient motion. These poor quality data components were either discarded or were not reconstructed because the data can only lead to poor reconstruction outcomes, which will affect the estimate of parameters in other regions as well. This shows that the accuracy of the reconstruction depends strongly on the capability to remove the artifacts.

Our reconstruction algorithm is convergence. The reconstruction outcomes using initial values taken from the estimated metabolite intensities, obtained from the zero-filled reconstruction, are compared to the reconstruction outcomes from using zero as initial values. Our proposed algorithm generates consistent outcomes from both estimations after certain iterations. This has shown the convergence of the algorithm. The modified EM optimization algorithm was typically run for 10-12 hours although no significant change 
in image quality was observed after approximately 5 hours. However, using the estimated metabolite intensities as the initial values is an efficient way to reduce the heavy computation for $3 \mathrm{D}$ reconstruction.

Some problems with the reconstruction are that the reconstruction can be affected by distorted signals in the in vivo whole brain volumetric MRSI, and can be affected by some degree of mis-registration between the MRSI and the MRI data. Also, note that the signals used here are in fact the combination of the phase array coil data sets, which could be another factor for estimate errors; however, such data are still preferred in comparison to the lower quality data from a single-head coil because the use of a phase-array coil takes advantage of the advanced acquisition technique [26]. In addition, the large MRSI dataset has made the reconstruction vulnerable to errors in estimation because large amount of parameters to be estimated. Although the number of parameters was reduced in our model, we still have a significant number of parameters to be estimated.

\section{CONCLUSIONS}

In this paper, a polynomial fitting improved Bayesian reconstruction method is proposed to reconstruct whole brain metabolite images for volumetric MRSI. It aims to simultaneously reduce slice thickness and increase the resolution of the metabolite images in all spatial directions. This method uses prior partial tissue concentration information of brain structure acquired from the co-registered MRI images to reduce the reconstruction errors resulted from the partial volume metabolite contributions. The polynomial fitting method is used to reduce the blocky patch phenomena caused by the modified EM algorithm. Our method combing the modified EM algorithm and the polynomial fitting method has been able to achieve resolution enhanced metabolic images; therefore, the quality of the reconstructed metabolite images is further improved.

\section{CONFLICT OF INTEREST}

The authors confirm that this article content has no conflicts of interest.

\section{ACKNOWLEDGEMENTS}

This work was supported by UNC Fayetteville State University Center of Defense and Homeland and Security (CDHS) Title III Activity, and a PHS grant NS041946. The authors would like to thank Dr. Claudia Domenig for acquiring the volumetric data for our processing.

\section{REFERENCES}

[1] Bao Y, Maudsley A. Improved Reconstruction for MR Spectroscopic Imaging. IEEE Trans Med Imaging 2007; 26(5):686-95.

[2] Soher BJ, Young K, Govindaraju V, Maudsley A. Automated spectral analysis III: application to in vivo proton MR spectroscopy and spectroscopic imaging. Magn Reson Med 1998; 40(6): 822-31.
[3] Liang ZP, Boada FE, Constable RT, Haacke EM, Lauterbur PC, Smith MR. Constrained reconstruction methods in MR imaging. Rev Magn Reson Med 1992; 4(2): 67-186.

[4] Wear KA. Advanced methods in spectroscopic imaging. In: Yan H, editor. Signal processing for magnetic resonance imaging and spectroscopy. New York: Marcel Dekker 2002.

[5] Plevritis SK, Macovski A. MRS imaging using anatomically based kspace sampling and extrapolation. Magn Reson Med 1995; 34: 686-93.

[6] Hu X, Patel MS, Garwood M, et al. Reduction of lipid contamination in proton chemical shift imaging by a Bayesian reconstruction. Proceedings of the International Society for Magnetic Resonance in Medicine 1992: p. 4253

[7] Spielman D, Webb P, Macovski A. A statistical framework for in vivo spectroscopic imaging. J Magn Reson 1988; 79(1): 66-77.

[8] $\mathrm{Hu}$ X, Levin DN, Lauterbur PC, Spraggins T. SLIM: Spectral localization by imaging. Magn Reson Med 1988; 8: 314-22.

[9] Liang ZP, Lauterbur PC. A generalized series approach to MR spectroscopic imaging. IEEE Trans Med Imaging 1991; 10: 132-7.

[10] Von Kienlin M, Mejia R. Spectral localization with optimal pointspread function. J Magn Reson 1991;94: 268-87.

[11] Bashir A, Yablonskiy DA. Natural linewidth chemical shift imaging (NL-CSI). Magn Reson Med 2006; 56(1): 7-18.

[12] Duijn JH, Matson GB, Maudsley A, Weiner MW. 3D phase encoding $1 \mathrm{H}$ spectroscopic imaging of human brain. Magn Reson Imaging 1992; 10: 315-9.

[13] Posse S, DeCarli C, Le Bihan D. Three-dimensional echo-planar MR spectroscopic imaging at short echo times in the human brain. Radiology 1994; 192: 733-8.

[14] Adalsteinsson E, Irarrazabal P, Spielman DM, Macovski A. Threedimensional spectroscopic imaging with time-varying gradients. Magn Reson Med 1995; 33: 461-6.

[15] Ebel A, Maudsley A. Improved spectral quality for 3D MR spectroscopic imaging using a high spatial resolution acquisition strategy. Magn Reson Imaging 2003; 21(2): 113-20.

[16] Haldar JP, Hernando D, Song SK, and Liang ZP. Anatomically constrained reconstruction from noisy data. Magn Reson Med 2008; 59: 810-8.

[17] Kim H, Catana C, Ratai EM, et al. Serial MR Spectroscopy Reveals a Direct Metabolic Effect of Cediranib in Glioblastoma Cancer Res 2011; 71(11): 3745-52.

[18] Bolan PJ, Meisamy S, Baker EH, et al. In vivo quantification of choline compounds in the breast with $1 \mathrm{H}$ MR spectroscopy. Magn Reson Med 2003; 50(6): 1134-43.

[19] Chen JH, Mehta RS, Vaea Baek HM, et al. Clinical characteristics and biomarkers of breast cancer associated with choline concentration measured by 1H MRS. NMR Biomed 2011; 24(3): 316-2

[20] Kelm BM, Kaster FO, Baek, HM, et al. Using spatial prior knowledge in the spectral fitting of MRS images. NMR Biomed 2012; 25(1): 1-13.

[21] Kornak J, Young K, Soher BJ, et al. Bayesian k-Space-Time Reconstruction of MR Spectroscopic Imaging for Enhanced Resolution. IEEE Trans Med Imaging 2010; 29(7): 1333-50.

[22] Dong Z, Peterson B. Spectral Resolution Amelioration by Deconvolution (SPREAD) in MR Spectroscopic Imaging. J Magn Reson Imaging 2009; 29(6): 1395-405.

[23] Eslami, R, Mathews, J. Robust reconstruction of MRSI data using a sparse spectral model and high resolution MRI priors. IEEE Trans Med Imaging 2010; 29(6): 1297-309.

[24] Statistical Parametric Mapping. Available from http://www.fil.ion.ucl.ac.uk/spm/

[25] Baker, EH, Basso, G, Barker, PB, Smith, MA, Bonekamp, D, Horská, A. Regional apparent metabolite concentrations in young adult brain measured by (1) H MR spectroscopy at 3 Tesla, J Magn Reson Imaging 2008; 27(3): 489-99.

[26] Zhu, X, Ebel, A, Ji, JX, Schuff, N. Spectral phase-corrected GRAPPA reconstruction of three-dimensional echo-planar spectroscopic imaging (3D-EPSI). Magn Reson Med 2007; 57(8):815-20.

(C) Bao and Maudsley; Licensee Bentham Open.

This is an open access article licensed under the terms of the Creative Commons Attribution Non-Commercial License (http://creativecommons.org/licenses/by-nc/3.0/) which permits unrestricted, non-commercial use, distribution and reproduction in any medium, provided the work is properly cited. 